\title{
AMENDMENTS
}

\section{Author Correction: A genome-wide association study with 1,126,563 individuals identifies new risk loci for Alzheimer's disease}

Douglas P. Wightman (D), Iris E. Jansen, Jeanne E. Savage (D), Alexey A. Shadrin D, Shahram Bahrami, Dominic Holland, Arvid Rongve (D), Sigrid Børte (D), Bendik S. Winsvold (D), Ole Kristian Drange, Amy E. Martinsen, Anne Heidi Skogholt, Cristen Willer (D), Geir Bråthen D, Ingunn Bosnes, Jonas Bille Nielsen, Lars G. Fritsche (D), Laurent F. Thomas (D), Linda M. Pedersen Di, Maiken E. Gabrielsen, Marianne Bakke Johnsen, Tore Wergeland Meisingset, Wei Zhou (D, Petroula Proitsi (D), Angela Hodges (D), Richard Dobson (D), Latha Velayudhan (D), Karl Heilbron, Adam Auton, 23andMe Research Team*, Julia M. Sealock (D), Lea K. Davis (D), Nancy L. Pedersen, Chandra A. Reynolds (D), Ida K. Karlsson, Sigurdur Magnusson D, Hreinn Stefansson (D), Steinunn Thordardottir, Palmi V. Jonsson, Jon Snaedal, Anna Zettergren (D), Ingmar Skoog, Silke Kern, Margda Waern, Henrik Zetterberg, Kaj Blennow, Eystein Stordal (D), Kristian Hveem, John-Anker Zwart (D), Lavinia Athanasiu, Per Selnes, Ingvild Saltvedt (D), Sigrid B. Sando, Ingun Ulstein, Srdjan Djurovic (D), Tormod Fladby DD, Dag Aarsland, Geir Selbæk (D), Stephan Ripke (D), Kari Stefansson (D), Ole A. Andreassen (D) and Danielle Posthuma (D)

Correction to: Nature Genetics https://doi.org/10.1038/s41588-021-00921-z, published online 7 September 2021.

In the version of this Article initially published, the author list and affiliations were incomplete. Karl Heilbron and Adam Auton, both with 23andMe, Inc., have been added to the author list. Further, the location for the 23andMe Research Team, now reading "Sunnyvale, CA" initially appeared as Mountain View, CA. The changes have been made to the online version of the paper.

${ }^{\star}$ A list of authors and their affiliations appears online.

Published online: 12 November 2021

https://doi.org/10.1038/s41588-021-00977-x

() The Author(s), under exclusive licence to Springer Nature America, Inc. 2021

\section{Author Correction: A high-resolution HLA reference panel capturing global population diversity enables multi-ancestry fine-mapping in HIV host response}

Yang Luo (D), Masahiro Kanai (D), Wanson Choi, Xinyi Li, Saori Sakaue (D), Kenichi Yamamoto, Kotaro Ogawa, Maria Gutierrez-Arcelus (D), Peter K. Gregersen (D), Philip E. Stuart (D), James T. Elder (D), Lukas Forer D, Sebastian Schönherr, Christian Fuchsberger (D), Albert V. Smith (D), Jacques Fellay (D), Mary Carrington (D), David W. Haas, Xiuqing Guo, Nicholette D. Palmer (D), Yii-Der Ida Chen, Jerome I. Rotter (D), Kent D. Taylor (D), Stephen S. Rich (D), Adolfo Correa (D), James G. Wilson, Sekar Kathiresan, Michael H. Cho (D), Andres Metspalu D, Tonu Esko D, Yukinori Okada (D), Buhm Han D, NHLBI Trans-Omics for Precision Medicine (TOPMed) Consortium*, Paul J. McLaren (iD and Soumya Raychaudhuri (D)

Correction to: Nature Genetics https://doi.org/10.1038/s41588-021-00935-7, published online 5 October 2021.

In the version of this Analysis initially published, author Buhm Han was missing an affiliation. In addition to the Interdisciplinary Program in Bioengineering, Seoul National University, Seoul, South Korea, his affiliations should also have included the Department of Biomedical Sciences, Seoul National University College of Medicine, Seoul, South Korea.

The change has been made to the online version of the article.

${ }^{\star}$ A list of authors and their affiliations appears online.

Published online: 2 November 2021

https://doi.org/10.1038/s41588-021-00979-9

(c) The Author(s), under exclusive licence to Springer Nature America, Inc. 2021 